\title{
Energy Estimation of Distributed Phase-shift Beamforming
}

\author{
Viktor Černý \\ Dept. of Computer Science and Engineering, Faculty of Electrical Engineering, Czech Technical University, Technická 2, \\ 16627 Praha, Czech Republic
}

Corresponding author: cernyvi2@fel.cvut.cz

\begin{abstract}
Ad-hoc and sensor networks are composed of small, low-power devices, which can connect between themselves without the help of an infrastructure. Research in this area has been both extensive and intensive and is still very far from exhaustion.

Our work in this area is aimed at developing a new type of communication between groups of modules capable of connecting clusters (groups) of devices which are separated by distances greater than the maximum transmission range of the devices themselves, without the help of relays or signal repeaters. In this paper we study the energy requirements for bidirectional communication between two clusters separated by a distance greater than the maximum transmission range of the modules, in the classic way (with the use of repeaters or relays) and by applying distributed phase-shift beamforming.
\end{abstract}

Keywords: ad-hoc networks, beamforming, phase-shift, distributed.

\section{Introduction}

Phase-shift beamforming is a method extensively used in radio networks, in which devices are equipped with multiple antennas. By injecting in each antenna element a signal slightly shifted in phase (delayed), because the signals travel slightly different distances towards the receiver, for a favourable phase combination, the two signals will meet at the receiver in phase and thus, will have energy higher than each of the signals taken separately. Such antenna systems (or antenna arrays) have been named "smart antennas" because, based on this method, the radiation pattern of the transmitter antenna array can be modified making its signal stronger or weaker in desired directions - without physically orienting the device, just by modifying the phase of the signals coming to each antenna element.

The case studied in this paper is of modules that are not equipped with antenna arrays - each device has only one single antenna. In order to be able to use the method of phase-shift beamforming, multiple devices have to share the same information to be sent and be able to synchronise the transmitted signals precisely. Such a mechanism has already been proposed in [6], [7], [8] and [1], however these papers ignore the fact that crystals are not perfect and phase synchronisation between the modules is lost due to the clock being slightly faster at one module than the other.

Our aim is briefly to present a method for synchronizing the transmitted signals, simpler than the method in [3], and to evaluate the energy requirements for bidirectional communication between two clusters of modules (separated by a distance greater than their

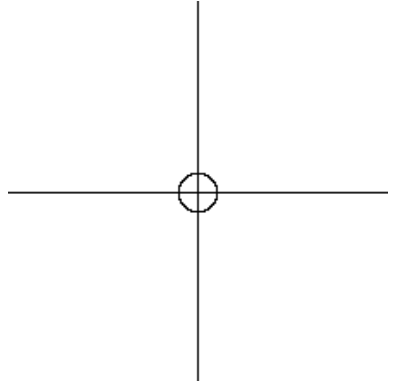

(a)

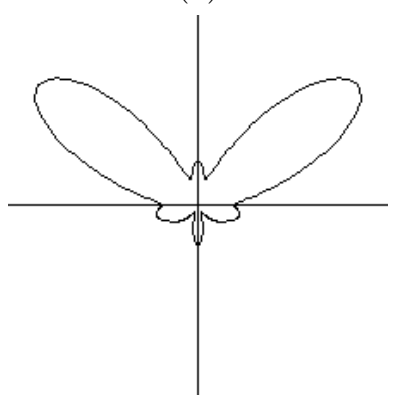

(c)

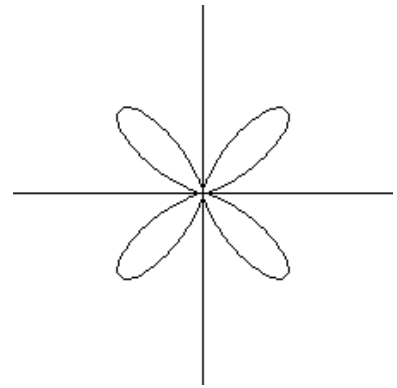

(b)

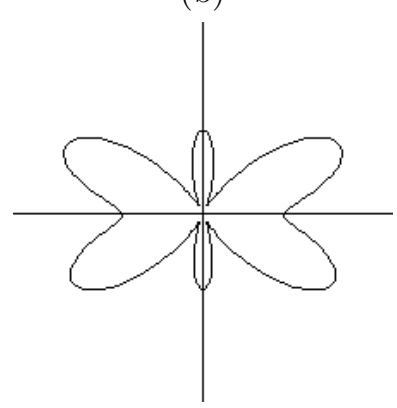

(d)
Figure 1: Different radiation patterns corresponding to different phase combinations of the signals injected into the elements of an antenna array.

maximal transmission range) in the method proposed here and in the classic way of installing repeaters (relays) between two clusters.

This paper is structured as follows: the second section presents the distributed phase-shift beamforming mechanism and the proposed device, the third section is a theoretical approach towards the required energy estimations, the fourth section contains the experi- 


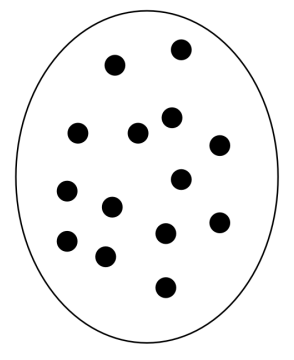

$\mathrm{K}_{1}$

(a)

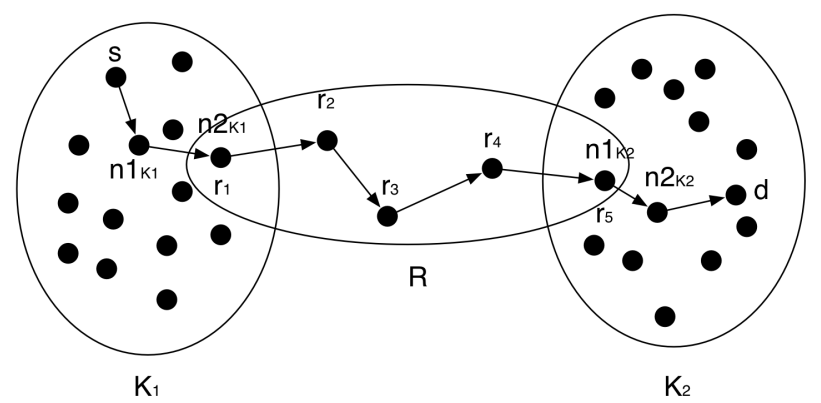

(b)

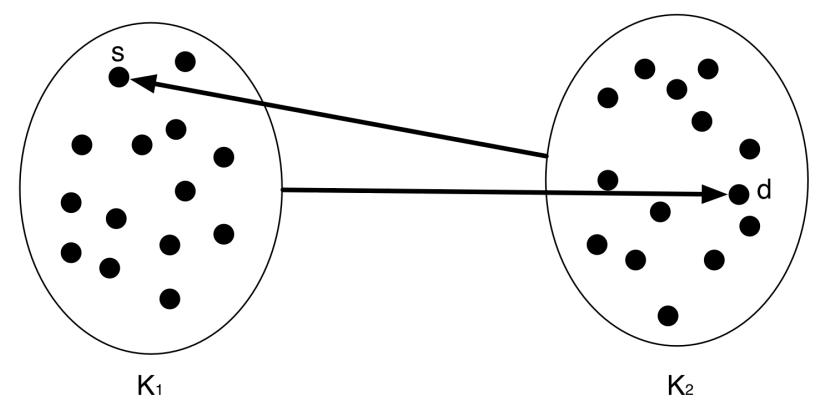

(c)

Figure 2: (a) A network divided into two clusters. (b) The two clusters can be connected by installing repeaters. (c) The two clusters can be connected by distributed phase-shift beamforming.

ments and the results and the paper is concluded in the fifth section, where future work is also proposed.

\section{Distributed Phase-Shift Beamforming}

There are four types of radio communications from the channel perspective: SISO, SIMO / MOSI, MISO / SOMI and MIMO. SISO (Single Input — Single Output) is the classic type - one antenna at the transmitter, one at the receiver. SIMO / MOSI (Single Input — Multiple Output) works at the basis of signal processing at the receiver; the transmitter is equipped with only one antenna and the receiver with multiple antennas. Noise affects the antennas at the receiver slightly differently and, with the use of signal processing, the useful signal can be sepa- rated from the noise. MISO (Multiple Input - Single Output) describes communications where the transmitter has multiple antennas, regulated by different initial phases, such that the signals coming from these antennas superimpose at the receiver antenna, thus creating a stronger signal. MIMO (Multiple Input Multiple Output) combines the properties of SIMO and MISO to present this technique here would be both space-consuming and beyond the scope of the paper.

To exemplify the properties of beamforming, let us consider four antennas $A_{1}, A_{2}, A_{3}$ and $A_{4}$ symmetrically placed at coordinates $(x, y): A_{1}(-\lambda / 2,0)$, $A_{2}(\lambda / 2,0), A_{3}(0,-\lambda / 2)$ and $A_{4}(0, \lambda / 2)$ and acting as an antenna array. Each antenna is assumed to have a perfectly circular radiation pattern in the horizontal plane, as in Fig. 1a ( $\lambda$ is the wavelength of the signal). If all the antennas are fed identical and in-phase signals, the radiation pattern of the whole array becomes as in Fig. 1b. If the signal injected in antenna $A_{4}$ is dephased at $-90^{\circ}$ and at $+180^{\circ}$ respectively taking as a reference the other three identical remaining signals, the radiation pattern of the array becomes as depicted in Fig. 1c and Fig. 1d respectively. In conclusion, we can manipulate the radiation pattern of a transmitter equipped with an antenna array by altering the initial phases of the signals injected into the element antennas composing the array.

Let us now consider an ad-hoc network where all the modules are randomly placed over a $2 \mathrm{D}$ surface and each module is equipped with only one single antenna. Let us also assume that, due to the random placement of the modules, two clusters are formed, without the possibility of direct communication between them, as in Fig. 2a. There are three ways to interconnect the devices by increasing the transmission power (this will drain the batteries faster and can be limited by the hardware of the transceiver), by using higher gain antennas (sometimes unsuitable because it will lead to physically bigger antennas) or by placing repeaters between the two clusters, as in Fig. 2 b.

We are currently developing a fourth way for the clusters to interconnect: the modules of one cluster will beamform their signal thus creating a virtual transmitter whose power will be sufficient to transmit directly to the other cluster - as in Fig. 2c.

If we assume the network presented in Fig. 2b, where the network is formed by two disjoint clusters $K_{1}$ and $K_{2}$, connected by a relay set $R$, the conditions for a connected network are: $R \neq \emptyset, R \cap K_{1} \neq \emptyset$ and $R \cap K_{2} \neq \emptyset$. Assuming a source module $s \in K_{1}$ and a destination module $d \in K_{2}$, connected by a "best route" discovered by a routing algorithm of some sort, as in Fig. 2b, then for each bidirectional communication between $\mathrm{s}$ and $\mathrm{d}$, in order to have reliable communication, any repeater $r \in R$ has to perform four steps: 
1. receive the data packet from the previous module;

2. transmit the data packet to the next module;

3. receive acknowledgement from the next module;

4. transmit the acknowledgement to the previous module.

In the same time, the end devices performed only two operations: $s$ transmitted a data packet and received an ACK, while $d$ received a data packet and sent an ACK.

In conclusion, because the number of operations performed by each relay is greater than the number of operations performed by each end device, we can expect that the batteries will exhaust at the relays sooner than at the end devices. In order to prevent this situation, we can install batteries with higher capacities at the relays, or we can increase the number of relay modules. There are however scenarios in which this improvement is impossible: for example, when there is a river in the area when the relay modules should be installed. In this case, the clusters are permanently disconnected.

By applying distributed phase-shift beamforming, $s$ will broadcast the data packet to its neighbours, and then the whole group of modules will transmit the data packet directly to $d$ in a synchronized manner. In order to reply, $d$ will broadcast its ACK locally to its own neighbours and then the whole group of modules will transmit in the ACK a synchronized manner directly to $s$ - as in Fig. 2c.

It is beyond the scope of this paper to present how the different modules will know how much to delay (dephase) the signals in order to superimpose correctly at the receiver - the cluster discovery mechanism has been studied, and the results were published in [5]. We can assume that in order to send a message from s to d, each module $m \in K_{1}$ has a precise phase delay $\Delta \phi_{m}$ which must be used so that cluster $K_{1}$ can transmit to d properly; similarly for the cluster $K_{2}$ in order to have communication from $d$ back to $s$.

The difficulty with this mechanism consists in maintaining the constant phase delay between different modules: the oscillator crystals are not identical, and even minute imperfections, over long periods of time, will lead to the loss of precise phase delays. One solution might be to repeat the phase discovery algorithm presented in [5], however this is not feasible due to its exponential complexity. Another solution that we have employed is if module $s$ is capable of transmitting simultaneously on two frequencies: $f_{d}$ - on which data is sent, and $f_{s}$ — which is a plain sine wave used for synchronization. Synchronization frequency $f_{s}$ was chosen $t$ times higher than data frequency $f_{d}$, where $t$ is a constant.

Thus, module $s$ is responsible for broadcasting information to its neighbours and for maintaining the synchronization of its neighbours during beamformed transmission to $d$. Each neighbour of $m$ of $s$ receives the synchronization signal $f_{s}$. Through a phase-locked loop it locks its own data signal $f_{d}$ and then adds to it the corresponding phase delay $\Delta \phi_{m}$. In this manner, the signals coming to $d$ from all the modules in $K_{1}$ will meet in phase and thus their power will increase, assuring transmission beyond the maximum range of any individual module separately.

The internal architecture of the module is presented in Fig. 3 and it is currently patent pending, under Patent Application, Reg. No. 2011-25254 / 2011-785, 2011. The components are the following:

1. Power supply - provides electric power to the active components.

2. Internal logic unit - performs the main tasks of the module (initiate phase search, dictates the regime based on channel access methods, computes the phase increment, etc.).

3. Carrier generator - generates two in-phase synchronised sine waves: one wave has frequency $f_{d}$ and is the data carrier, the other has frequency $f_{s}=t \stackrel{\circ}{\mathrm{f}} f_{d}$ and is the synchronisation signal. This can be achieved for example by involving a crystal capable of generating $f_{s}$ and then a frequency divider which generates $f_{d}$ from $f_{s}$, by dividing it to $t>1$.

4. Transceiver - using a modulation technique, this is responsible for sending and receiving information on the data wave.

5. Phase synchroniser - this is in essence a phaselocked loop (PLL) capable of synchronising two waves.

6. Phase delayer - delays the phase of a signal by a given amount.

7. Mode switch - commutes the module from one regime (or mode of operation) to another.

8. Antenna - the antenna used for transmitting and receiving; it must allow frequencies $f_{d}$ and $f_{s}$ to pass through.

\section{$3 \quad$ Energy Estimation}

Case A. Let us start with classic bidirectional storeand-forward communication between two radio modules $M_{1}$ and $M_{2}$, separated by distance $D$, in free space, as in Fig. 4a. For a classic bidirectional communication, the minimal number of packets is 4: DATA $\left(M_{1} \rightarrow M_{2}\right)$, ACK $\left(M_{2} \rightarrow M_{1}\right)$, DATA $\left(M_{2} \rightarrow M_{1}\right)$ and $\operatorname{ACK}\left(M_{1} \rightarrow M_{2}\right)$. We can assume that the size of the ACK packet is $k$ times larger than the size of the DATA packet, where $0<k<1$ and we note that 


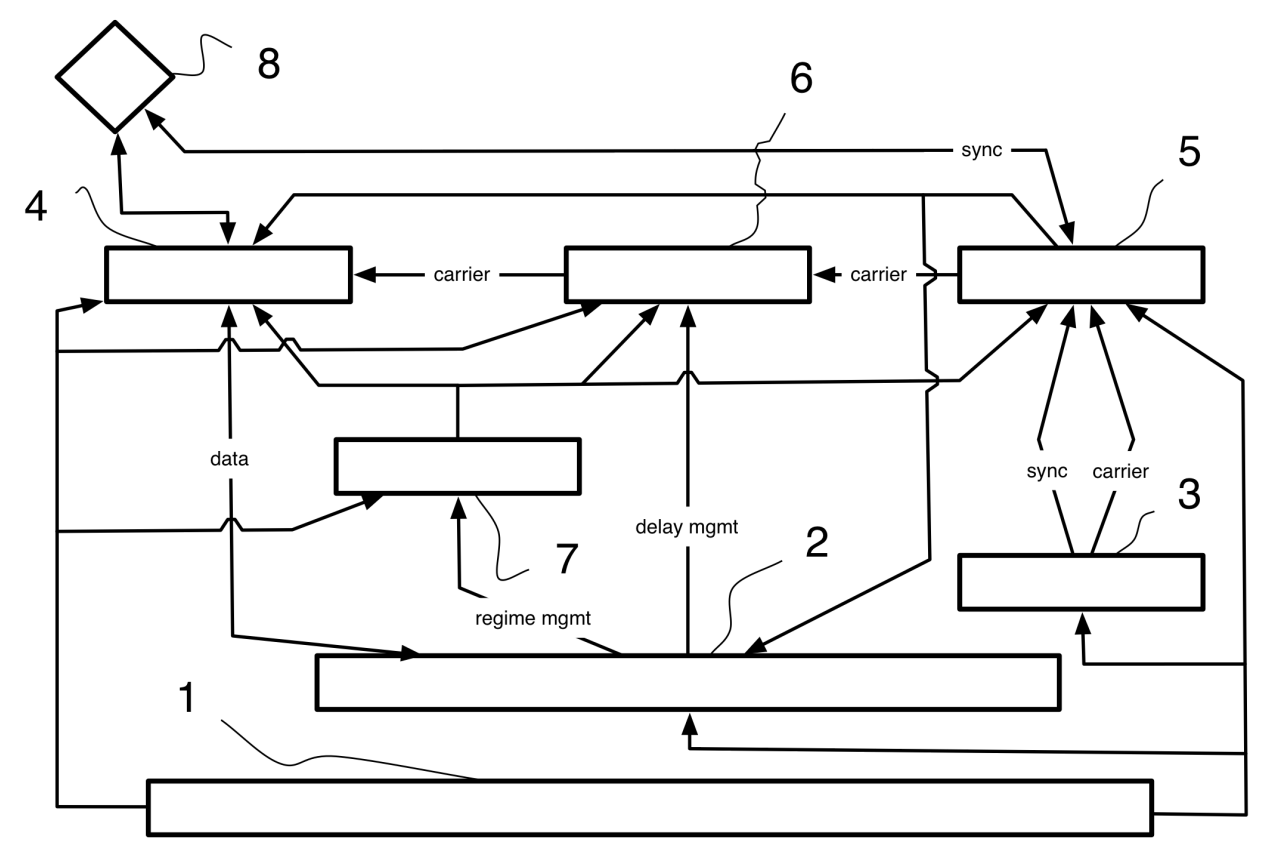

Figure 3: Device capable of distributed phase-shift beamforming. The full functioning of the device is described in the Patent Application Form [4].

sizeof $(\mathrm{ACK})=k$ sizeof(DATA). From the laws of electromagnetic propagation in free space (FSPL) it can be seen that the received power decreases with the distance squared. For energy estimation, because the methods are compared with each other, it is safe to assume (in order to simplify the calculus) that the antennas have no gain. In the same manner, we consider the receiving operation as being totally passive (no energy required). In this case, in milliwatts, according to FSPL we can write that $P_{R} \sim \frac{P_{T}}{D^{2}}$. The received power is proportional to the transmitted power and is inreversely proportional to the squared distance. Thus, the energy $E$ required to transmit a data packet over distance $D$ can be written as:

$$
E \sim D^{2} \operatorname{sizeof}(\mathrm{DATA})
$$

and we can choose the constant to balance this inequality as $C$ (dependent on the antenna gains, apertures, minimal required power at the receiver, the environment of propagation and sizeof(DATA)), thus:

$$
E(\mathrm{DATA})=C D^{2} .
$$

In this case, $E(\mathrm{DATA})=C D^{2}=E_{D}$ and $E(\mathrm{ACK})=k C D^{2}=E_{A}=k E_{D}$. The total energy required for the minimal bidirectional communication becomes:

$$
E_{\text {tot }}=2\left(E_{D}+E_{A}\right)=2 C D^{2}(1+k) .
$$

The energy need per single module is:

$$
E_{\text {mod }}=\left(E_{D}+E_{A}\right)=C D^{2}(1+k) .
$$

Case B. Let us now consider the same case, though now between $M_{1}$ and $M_{2}$ there is a repeater $R$, placed at distances $D_{1}$ and $D_{2}$ from $M_{1}$ and $M_{2}$ respectively, as in Fig. 4b. For duplex store-and-forward communication, the minimal number of data packets is 8 : $\operatorname{DATA}\left(M_{1} \rightarrow R\right), \operatorname{DATA}\left(R \rightarrow M_{2}\right), \operatorname{ACK}\left(M_{2} \rightarrow R\right)$, $\operatorname{ACK}\left(R \rightarrow M_{1}\right), \operatorname{DATA}\left(M_{2} \rightarrow R\right), \operatorname{DATA}\left(R \rightarrow M_{1}\right)$, $\operatorname{ACK}\left(M_{1} \rightarrow R\right)$ and $\operatorname{ACK}\left(R \rightarrow M_{2}\right)$.

In order to send one data packet from $M_{1}$ to $M_{2}$ through $R$, the total energy required will be, according to $(2)$ :

$$
E=E_{1}+E_{2}=C D_{1}^{2}+C D_{2}^{2}=C\left(D_{1}^{2}+\left(1-D_{1}\right)^{2}\right)
$$

It can be seen that $E$ has its minimal value when $D_{1}=D_{2}=D$, thus the most efficient way (from the energy point of view) to place a repeater is to place it in the middle of the distance between the modules. In this case, we obtain: $E(\mathrm{DATA})=C\left(\frac{D}{2}\right)^{2}=\frac{C D^{2}}{4}=$ $\frac{E_{D}}{4}$ and in the same manner: $E(\mathrm{ACK})=k C\left(\frac{D}{2}\right)^{2}=$ $\frac{E_{A}}{4}=\frac{k E_{D}}{4}$. The total energy need is:

$$
\begin{aligned}
& E_{t o t}=4(E(\mathrm{DATA})+E(\mathrm{ACK})) \\
&=4\left(\frac{E_{D}}{4}+\frac{k E_{D}}{4}\right)=C D^{2}(1+k) .
\end{aligned}
$$

The energy drain per end-module is given by:

$$
E_{m o d}=E(\mathrm{DATA})+E(\mathrm{ACK})=\frac{1}{4} C D^{2}(1+k)
$$

and for the relay:

$$
E_{R}=2(E(\mathrm{DATA})+E(\mathrm{ACK}))=\frac{1}{2} C D^{2}(1+k) .
$$




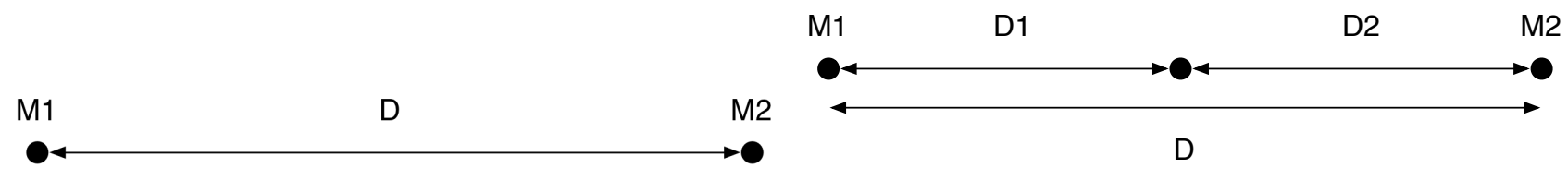

(a) Two modules.

(b) Two modules and one repeater.
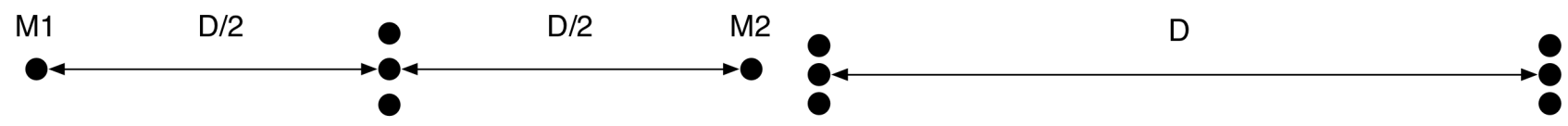

(c) Two modules and $N$ relays.

$M$ modules per cluster.

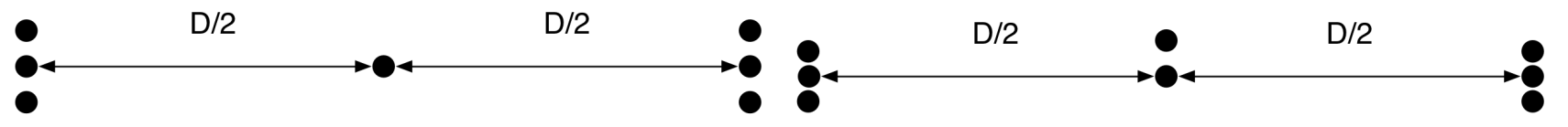

(e) $M$ modules per cluster and one relay.

(f) $M$ modules per cluster and $N$ relays.

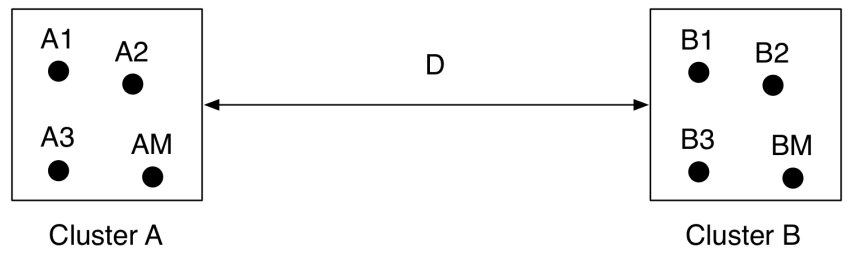

(g) $M$ modules per cluster, phase-shift beamforming.

Figure 4: Different module placements.

It can be seen that even in this most effective case, the need on the router is twice the need on any enddevice, thus the router will exhaust its battery twice as fast as any of the modules. However, if the router can allow for a battery twice the capacity of any end-module, this will compensate the higher power need.

Case C. If between the devices there are $N$ relays, as in Fig. $3.27 \mathrm{c}$, then the average power consumption becomes per end device (the same as in (7)):

$$
E_{\text {mod }}=E(\mathrm{DATA})+E(\mathrm{ACK})=\frac{1}{4} C D^{2}(1+k),
$$

and per repeater:

$$
E_{R}=2(E(\mathrm{DATA})+E(\mathrm{ACK}))=\frac{1}{2 N} C D^{2}(1+k) .
$$

The total energy need remains the same as in (6). Thus, installing more repeaters can compensate the rapid exhaustion of the battery seen in Case B.

Case D. Assuming the same topology as in Case A (Fig. 4a), though with $n$ data packets per end-device, then:

$$
E_{\text {tot }}=2 n C D^{2}(1+k),
$$

and the energy drain per end-module:

$$
E_{\text {mod }}=n C D^{2}(1+k) .
$$

Case E. Supposing the same topology as in Case B (Fig. 4b), though with $n$ data packets per end-device, we obtain:

$$
\begin{aligned}
E_{t o t} & =n C D^{2}(1+k), \\
E_{\text {mod }} & =\frac{n}{4} C D^{2}(1+k), \\
E_{R} & =\frac{n}{2} C D^{2}(1+k) .
\end{aligned}
$$

Case F. Assuming the same topology as in Case C (Fig. 4c), though with $n$ data packets per end-device, we obtain:

$$
\begin{aligned}
E_{t o t} & =n C D^{2}(1+k), \\
E_{\text {mod }} & =\frac{n}{4} C D^{2}(1+k), \\
E_{R} & =\frac{n}{2 N} C D^{2}(1+k) .
\end{aligned}
$$

Case G. Considering now $M$ modules per cluster, each generating $n$ data packets, as in Fig. $4 d$, then:

$$
\begin{aligned}
E_{\text {tot }} & =2 M n C D^{2}(1+k), \\
E_{\text {mod }} & =n C D^{2}(1+k),
\end{aligned}
$$

Case H. Considering now $M$ modules per cluster, each generating $n$ data packets and one repeater in 
the middle, as in Fig. 4e, then:

$$
\begin{aligned}
E_{t o t} & =M n C D^{2}(1+k), \\
E_{\text {mod }} & =\frac{n}{4} C D^{2}(1+k), \\
E_{R} & =\frac{M n}{2} C D^{2}(1+k) .
\end{aligned}
$$

We assumed for this case that distance $D$ is much greater than the distances between the modules of a cluster.

Case I. Considering now $M$ modules per cluster, each generating $n$ data packets and $N$ repeaters in the middle, as in Fig. 4f, then:

$$
\begin{aligned}
E_{t o t} & =M n C D^{2}(1+k), \\
E_{\text {mod }} & =\frac{n}{4} C D^{2}(1+k), \\
E_{R} & =\frac{M n}{2 N} C D^{2}(1+k) .
\end{aligned}
$$

Case J: Let us consider two clusters, separated by a distance $D$ that is much greater than the cluster size (a squared area $d \times d$ meters - Fig. 4g). Each cluster contains $M$ modules. Let us note the energy that a module of a cluster needs to broadcast a data packet to the other members of the same cluster as $E_{B D}$ and the energy that a module of a cluster needs to broadcast an acknowledgement packet to the other members of the same cluster as $E_{B A}$. Let us also assume that each module has equal participation in the cluster in spreading the load, in beamforming and generating data traffic. We can then write that:

- the energy to broadcast data in the cluster is $E_{B D}=s E_{D}$ and

- the energy to broadcast acknowledgement in the cluster is $E_{B A}=s E_{A}=k s E_{D}$.

The steps required for 1-packet two-way communication between modules $A_{1}$ and $B_{1}$ are:

1. module $A_{1}$ broadcasts data in cluster $A$ on frequency $f_{d}$, Fig. 5a;

2. cluster $A$ is synchronised by $A_{1}$ on frequency $f_{s}$ and in one step transmits data to module $B_{1}$, Fig. 5b;

3. module $B_{1}$ broadcasts an acknowledgement in cluster $B$ on frequency $f_{d}$, Fig. 5 c;

4. cluster $B$ is synchronised by $B_{1}$ on frequency $f_{s}$ and in one step transmits the acknowledgement to module $A_{1}$, Fig. 5 d;

5. module $B_{1}$ broadcasts data in cluster $B$ on frequency $f_{d}$, Fig. 5e;
6. cluster $B$ is synchronised by $B_{1}$ on frequency $f_{s}$ and in one step transmits data to module $A_{1}$, Fig. 5f;

7. module $A_{1}$ broadcasts the acknowledgement in cluster $A$ on frequency $f_{d}$, Fig. 5g;

8. cluster $A$ is synchronised by $A_{1}$ on frequency $f_{s}$ and in one step transmits the acknowledgement to module $B_{1}$, Fig. 5 h.

The energy requirements for each step are:

Step 1 - module $A_{1}$ broadcasts data in cluster $A$ on frequency $f_{d}$, Fig. 5a:

- at $A_{1}: E_{1}=E_{B D}=s E_{D}$;

- at other modules: 0 .

Step 2 - cluster $A$ is synchronised by $A_{1}$ on frequency $f_{s}$ and in one step transmits data to module $B_{1}$, Fig. 5b:

- at each $A_{i}: E_{2}=\frac{E(\mathrm{DATA})}{M}=\frac{C D^{2}}{M}$;

- $A_{1}$ has also to synchronise the cluster: $E_{3}=$ $E_{S D}=t E_{B D}$, where $t$ is a constant;

- at modules belonging to cluster $B: 0$.

Step 3 - module $B_{1}$ broadcasts the acknowledgement in cluster $B$ on frequency $f_{d}$, Fig. $5 \mathrm{c}$ :

- at $B_{1}: E_{4}=E_{B A}=s E_{A}$;

- at other modules: 0 .

Step 4 - cluster $B$ is synchronised by $B_{1}$ on the frequency $f_{s}$ and in one step transmits the acknowledgement to module $A_{1}$, Fig. 5 d:

- at each $B_{i}: E_{5}=\frac{E(\mathrm{ACK})}{M}=\frac{k C D^{2}}{M}$;

- $B_{1}$ has also to synchronise the cluster: $E_{6}=$ $E_{S A}=t E_{B A}$, where $t$ is a constant;

- at modules belonging to cluster $A$ : 0 .

Step 5 - module $B_{1}$ broadcasts data in cluster $B$ on frequency $f_{d}$, Fig. 5e:

- at $B_{1}: E_{7}=E_{B D}=s E_{D}$;

- at other modules: 0 .

Step 6 - cluster $B$ is synchronised by $B_{1}$ on frequency $f_{s}$ and in one step transmits data to module $A_{1}$, Fig. 5f:

- at each $B_{i}: E_{8}=\frac{E(\text { DATA })}{M}=\frac{C D^{2}}{M} ;$

- $B_{1}$ also has to synchronise the cluster: $E_{9}=$ $E_{S D}=t E_{B D}$, where $t$ is a constant;

- at modules belonging to cluster $A$ : 0 .

Step 7 - module $A_{1}$ broadcasts the acknowledgement in cluster $A$ on frequency $f_{d}$, Fig. $5 \mathrm{~g}$ : 


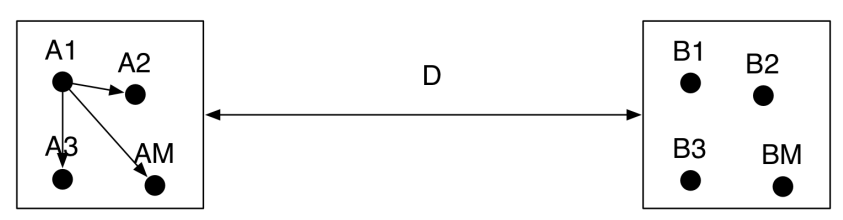

Cluster A

$A_{1}$ local DATA bcast.

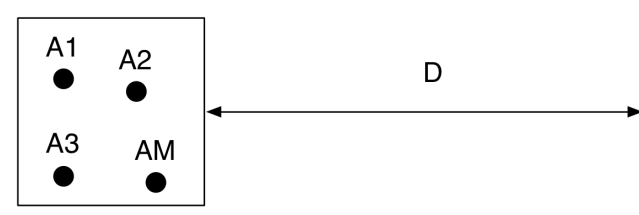

Cluster A

$B_{1}$ local ACK bcast.

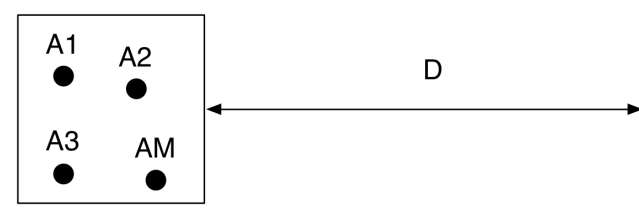

Cluster A

$B_{1}$ local DATA bcast.

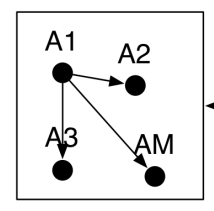

Cluster A

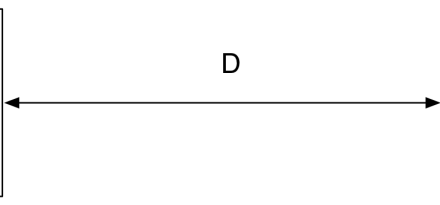

$A_{1}$ local ACK bcast.

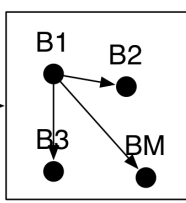

Cluster B

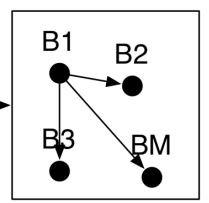

Cluster B

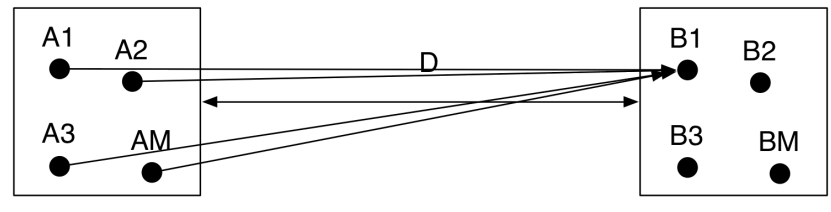

Cluster A

Cluster B

Beamformed DATA transmission $A \rightarrow B_{1}$.

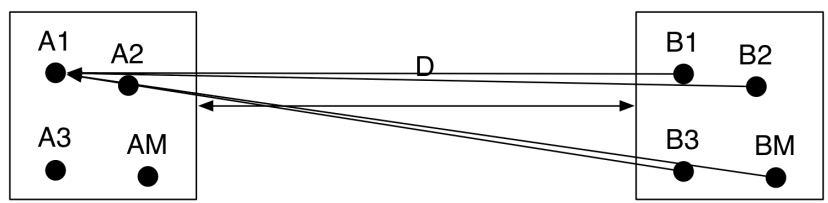

Cluster A
Beamformed ACK transmission $B \rightarrow A_{1}$.

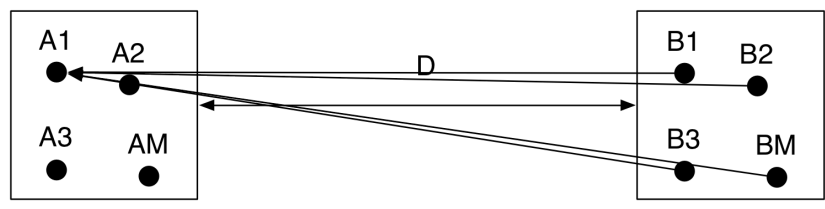

Cluster A

Cluster B

Beamformed DATA transmission $B \rightarrow A_{1}$.

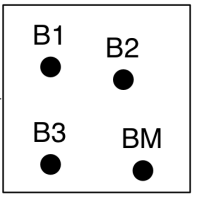

Cluster B

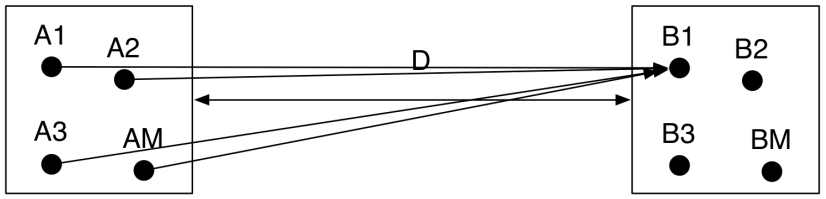

Cluster A
Cluster B
Beamformed ACK transmission $A \rightarrow B_{1}$.

Figure 5: Steps of a beamformed bidirectional communication.

- at $A_{1}: E_{10}=E_{B A}=s E_{A}$

- at other modules: 0 .

Step 8 - cluster $A$ is synchronised by $A_{1}$ on frequency $f_{s}$ and in one step transmits the acknowledgement to module $B_{1}$, Fig. 5 h:

- at each $A_{i}: E_{11}=\frac{E(\mathrm{ACK})}{M}=\frac{k C D^{2}}{M}$;

- $A_{1}$ also has to synchronise the cluster: $E_{12}=E_{S A}=t E_{B A}$, where $t$ is a constant;

- at modules belonging to cluster $B$ : 0 .

The total energy required by the whole network becomes the sum of $E_{1}$ to $E_{12}$, where some of the terms have to be multiplied by $M$ (the number of nodes):

$$
\begin{aligned}
E_{t o t}=2\left(E_{B D}\right. & +M \frac{C D^{2}}{M}+t E_{B D} \\
& \left.+E_{B A}+M k \frac{C D^{2}}{M}+t E_{B A}\right) .
\end{aligned}
$$

We have previously seen that $E_{D} \sim D^{2}$ and we noted $E_{D}=C D^{2}$. In the same manner, because the distances between modules belonging to the same cluster have the same order with cluster size $d$, we can write that $E_{B D} \sim d^{2}$ and $E_{B D}=C d^{2}$. Thus, by replacing these values into (25) we obtain:

$$
\begin{array}{r}
E_{\text {tot }}=2\left(C d^{2}+C D^{2}+t C d^{2}+k C d^{2}+k C D^{2}+t k C d^{2}\right) \\
=2 C(1+k)\left((1+t) d^{2}+D^{2}\right) .
\end{array}
$$

Energy need per device: for module $A_{1}: E_{A_{1}}=E_{1}+$ $E_{2}+E_{3}+E_{10}+E_{11}+E_{12}$ :

$$
\begin{array}{r}
E_{A_{1}}=C d^{2}+ \\
t C d^{2}+k C d^{2}+t k C d^{2}+\frac{C D^{2}}{M}(1+k) \\
=C(1+k)\left((1+t) d^{2}+\frac{D^{2}}{M}\right) ; \quad(27)
\end{array}
$$

for module $B_{1}: E_{B_{1}}=E_{4}+E_{5}+E_{6}+E_{7}+E_{8}+E_{9}$ :

$$
E_{B_{1}}=C(1+k)\left((1+t) d^{2}+\frac{D^{2}}{M}\right)=E_{A_{1}} ;
$$

for the other modules in cluster $A$ : $E_{C A}=E_{2}+E_{11}$ :

$$
E_{C A}=\frac{C D^{2}}{M}(1+k)
$$

for the other modules in cluster $B: E_{C B}=E_{5}+E_{8}$ :

$$
E_{C B}=\frac{C D^{2}}{M}(1+k)=E_{C A} .
$$


In conclusion:

- The total energy needed per network:

$$
E_{t o t}=2 C(1+k)\left((1+t) d^{2}+D^{2}\right)
$$

- The energy for devices which are data sources and sync sources:

$$
E_{\text {active }}=C(1+k)\left((1+t) d^{2}+\frac{D^{2}}{M}\right) .
$$

- The energy for devices which only help transmission:

$$
E_{\text {passive }}=\frac{C D^{2}}{M}(1+k) .
$$

Case K. Let us take the same topology as in Case $\mathrm{J}$, but this time each device generates $\mathrm{n}$ data packets. In this case, the average energy per device can be calculated in the following manner:

- We start by counting the data packets in the network: $2 M n$.

- In one cluster, each device is active for $n$ packets (its own packets) and passive for the remaining $(M-1) n$ packets. In total, each cluster is responsible for $M n$ packets (half of the total number of packets).

Thus: the total energy per network becomes:

$$
E_{t o t}=2 C M n(1+k)\left((1+t) d^{2}+D^{2}\right) ;
$$

the energy per device is $E_{\text {mod }}=n E_{\text {active }}+$ $(M-1) n E_{\text {passive: }}$

$$
E_{\text {mod }}=n C(1+k)\left((1+t) d^{2}+\frac{D^{2}}{M}\right) .
$$

Final conclusions. Let us compare the total energy requirements in cases K (general beamforming) and I (general relay):

$$
\begin{aligned}
& \frac{E_{t o t, \mathrm{~K}}}{E_{t o t, \mathrm{I}}}=\frac{2 C M n(1+k)\left((1+t) d^{2}+D^{2}\right)}{M n C D^{2}(1+k)} \\
&=4\left(1+(1+t)\left(\frac{d}{D}\right)^{2}\right)
\end{aligned}
$$

If $D \gg d$, then $\frac{E_{t o t, \mathrm{~K}}}{E_{t o t, \mathrm{I}}} \rightarrow 2$, meaning that the overall energy consumption beamforming presented in case $\mathrm{K}$ is twice as inefficient as relay transmission of the same order.
Let us now compare the energy requirements of end devices in cases $\mathrm{K}$ (general beamforming) and I (general relay):

$$
\begin{aligned}
& \frac{E_{\text {mod, } \mathrm{K}}}{E_{\text {mod }, \mathrm{I}}}= \\
& \frac{n C(1+k)\left((1+t) d^{2}+\frac{D^{2}}{M}\right)+(M-1) n \frac{C D^{2}}{M}(1+k)}{\frac{1}{4} n C D^{2}(1+k)} \\
& =4\left(1+(1+t)\left(\frac{d}{D}\right)^{2}\right) .
\end{aligned}
$$

If $D \gg d$, then $\frac{E_{m o d, \mathrm{~K}}}{E_{t o t, \mathrm{I}}} \rightarrow 4$. meaning that the enddevice energy consumption beamforming presented in case $\mathrm{K}$ is four times as inefficient as relay transmission of the same order.

Finally, let us now compare the energy requirement of end devices in case $\mathrm{K}$ (general beamforming) and the energy requirement of relays in case I (general relay):

$$
\begin{aligned}
& \frac{E_{\text {mod, } \mathrm{K}}}{E_{R, \mathrm{I}}}= \\
& \frac{n C(1+k)\left((1+t) d^{2}+\frac{D^{2}}{M}\right)+(M-1) n \frac{C D^{2}}{M}(1+k)}{\frac{M n}{2 n} C D^{2}(1+k)} \\
& =\frac{2 N}{M}\left(1+(1+t)\left(\frac{d}{D}\right)^{2}\right) .
\end{aligned}
$$

If $D \gg d$, then $\frac{E_{m o d, \mathrm{~K}}}{E_{t o t, \mathrm{I}}} \rightarrow \frac{2 N}{M}$. In this case, if $M=N$ (the number of end-devices is equal to the number of relays), the energy consumption in the case of beamforming per device is twice the energy consumption on a relay.

Proposition. In a clustered network, relaying is at limit twice more efficient for equivalent networks, per total power consumption and per end device. In environments in which relays cannot be installed or if the graph cut (of the network) contains a number of relays less than half of the number of end-devices, then the network connected through phase-shift beamforming will survive more than relaying

Proof. The proof has been already provided in a constructive way in this section.

Of course, the estimation here is an approximation, because we have ignored the small distance differences inside and outside the clusters. This however gives a better understanding of the quantitative improvements brought by phase-shift beamforming and in this way give a maximum theoretical limit. 


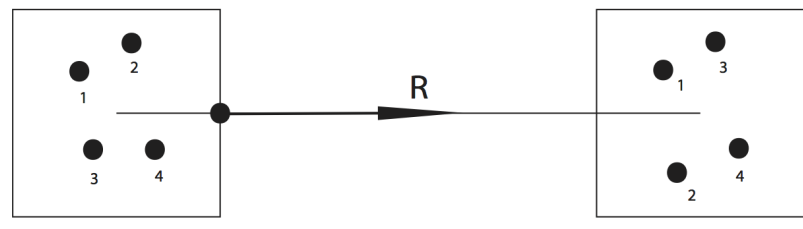

A

Figure 6: The relay module position is varied along the line uniting the centres of the two clusters.

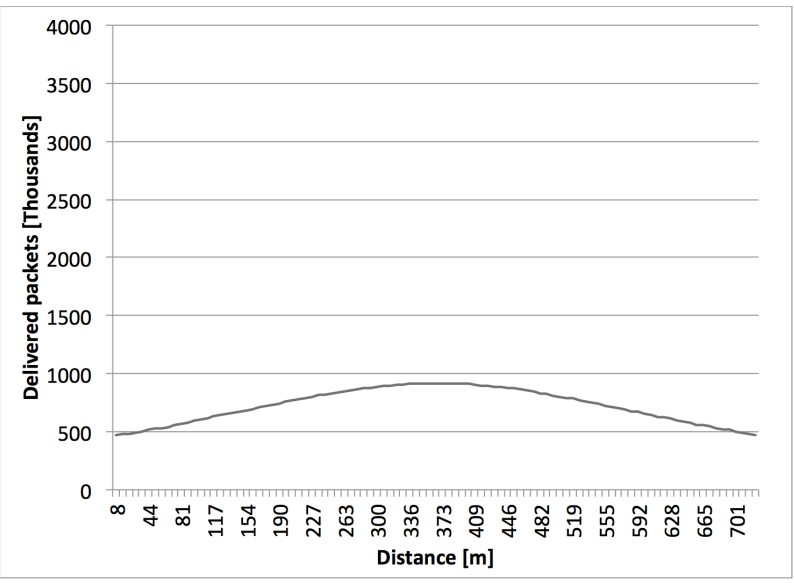

Figure 7: Packets delivered between clusters in both directions. The vertical axis represents the number of packets, and the horizontal axis represents the distance between the relay and the centre of cluster $A$ (in meters).

\section{Experiments and Results}

The simulations presented in this section are based on the following assumptions:

- a free-space propagation model (no obstacles for waves, no reflexions);

- battery capacity for each device: $200 \mathrm{mWh}$ (larger capacities are also permitted, but the simulation will last longer);

- two clusters, each containing 4 modules;

- squared cluster areas;

- random placement of modules in the cluster area;

- $P_{\min }=-75 \mathrm{dBm}$ (minimal power);

- data rate $D R=115200 \mathrm{bps}$;

- antenna gain: $1.5 \mathrm{dBi}$ isotropic (for the results presented here, isotropic antennas were used to show the contribution of the distributed phase-shift beamforming in a separate manner anisotropic antennas can also be simulated);

- data packet size: 128 B (the size of a ZigBee packet);

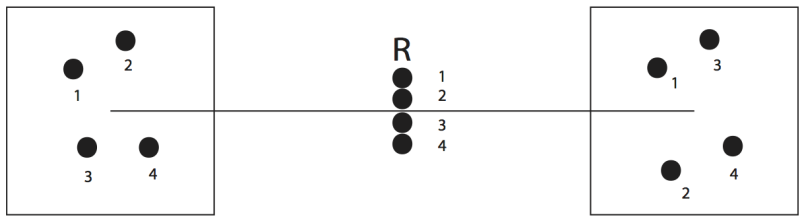

A

B

Figure 8: 1 to 4 relay nodes in between the clusters.

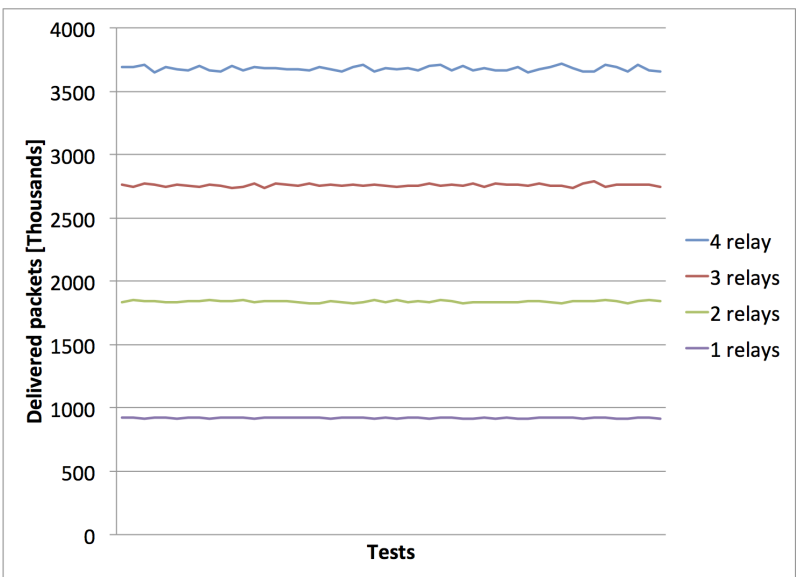

Figure 9: Packets delivered between clusters in both directions. The vertical axis represents the number of packets, and the horizontal axis is the number of tests performed.

- acknowledgement size: $12 \mathrm{~B}$;

- synchronization constant $t=2$;

- random traffic is generated from each cluster to the other cluster (random source module and random destination module, where the source and destination belong to different clusters);

- each presented result is the averaged value over 10 simulations;

- in the results, only the number of delivered packets is presented, because the time intervals are proportional to the number of packets.

\subsection{Experiment 1}

Two clusters of $10 \times 10 \mathrm{~m}$, containing 4 modules each, are placed $1000 \mathrm{~m}$ from each other. In between there is a single relay module. The relay is moved along the line which passes through the centres of each cluster from the proximity of the first cluster to the proximity of the second cluster (Fig. 6). The goal is to determine the number of packets and the time interval in which the network is functioning.

The result of this experiment is presented in Fig. 7, and it shows that the best result (the longest uptime) is achieved when the relay is placed in the middle of the distance between the two clusters. In all the 


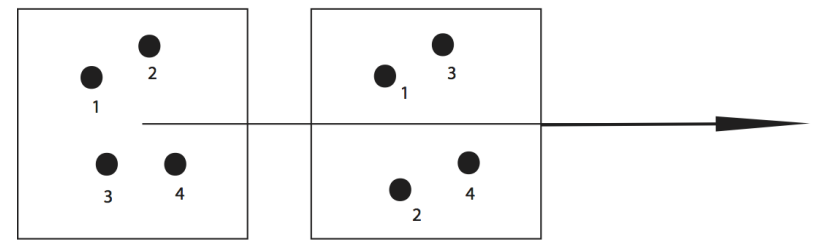

A

B

Figure 10: The $B$ cluster moves away from cluster A.

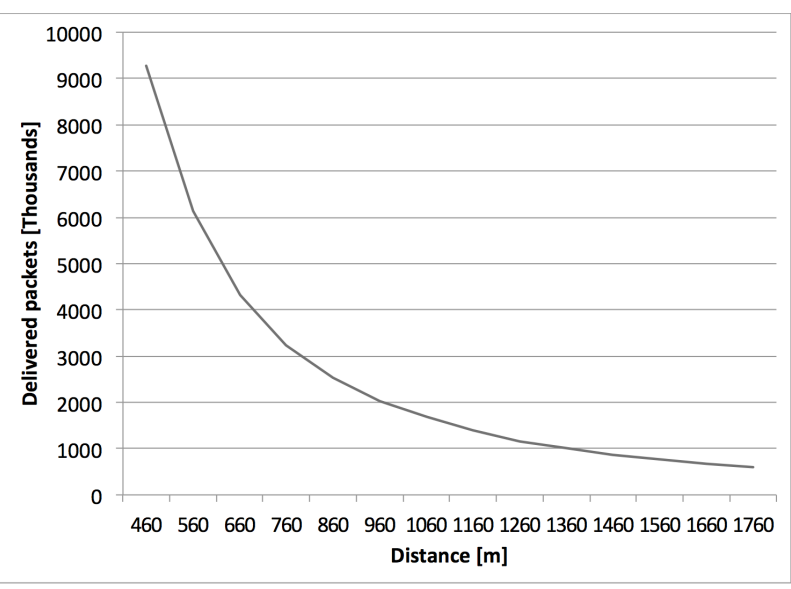

Figure 11: Packets delivered between clusters in both directions. The vertical axis represents the number of packets, and the horizontal axis represents the distance between the clusters (in meters).

tested cases, the clusters become disconnected due to exhaustion of the relay battery. The fact that the optimum is reached when the relay sits in the middle of the distance has been theoretically shown; the results match the prediction.

\subsection{Experiment 2}

Two clusters of $10 \times 10 \mathrm{~m}$, containing 4 modules each, are placed $1000 \mathrm{~m}$ from each other. In between there are 1 to 4 relay modules used in a round-robin fashion (Fig. 8). The goal is to determine the number of packets and the time interval in which the network is functioning.

The results depicted in Fig. 9 show a dramatic improvement as the number of relay modules increases. In all the cases the clusters become disconnected due to exhaustion of the relay battery. This leads to one conclusion: if relays can be installed, this is the preferred method.

\section{$4.3 \quad$ Experiment 3}

Two clusters of $10 \times 10 \mathrm{~m}$, containing 4 modules each, are placed $450 \mathrm{~m}$ from each other. Cluster $B$ is then moved along the axis that connects the centres of

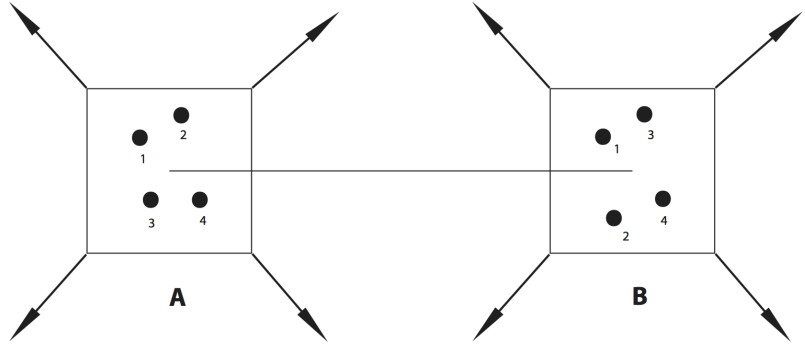

Figure 12: Increasing the cluster sizes.

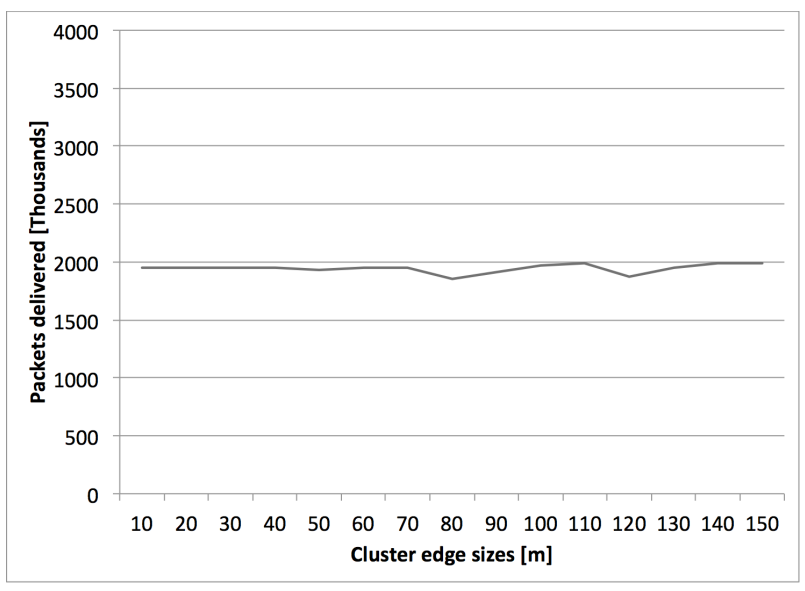

Figure 13: The dependency of the number of delivered packets on cluster size. On the vertical axis, the number of delivered packets; on the horizontal axis, the size of the clusters in meters.

the two clusters, away from cluster $A$, up to $2000 \mathrm{~m}$. There are no relay nodes, only phase-shift beamformed communication. The goal is to determine the number of packets and the time interval in which the network is functioning. (Fig. 10)

The results are depicted in Fig. 11, which shows how distance affects the overall number of delivered packets. The disconnection is caused by exhaustion of the batteries of either the source node or the destination node.

What is really interesting in this graph (Fig. 11) is that at a distance of $1000 \mathrm{~m}$ between the clusters, the number of acknowledged packets is approximately 3.7 million. By comparison, the best-case scenario for a single relay is just below one million. This proves that phase-shift beamforming can outperform classic relaying by a factor of 3 if the number of relays is very low.

\subsection{Experiment 4}

Two clusters of $10 \times 10 \mathrm{~m}$, containing 4 modules each, are placed $2000 \mathrm{~m}$ from each other. The size of each cluster is increased (from $10 \times 10 \mathrm{~m}$ to $10 \times 10 \mathrm{~m}$ ), while its centre remains on the same coordinates. This method proves the stability of phase-shift beamformed communication between clusters (Fig. 12). The results 
are depicted in Fig. 13, and show that the number of delivered packets is almost constant at around one million, which corresponds to the result obtained in Experiment 3 for the same distance between clusters.

\section{Conclusions and future work}

The work presented in this paper aimed to determine the advantages and disadvantages of using distributed phase-shift beamforming in comparison with classic repeater techniques for interconnecting two distant clusters of radio devices.

In this work certain energy consumptions were ignored, e.q. for the data processing before the energy is transmitted or after it is received. However, the expected power needs are constant for each received or processed data packet, depending only on the size of the respective packet. Because the results are presented both in absolute form and in relative form (beamformed towards a classic repeater), the influence of these constant power on the relative results is minimal.

The previous section has shown that, by comparing the worst result achievable through distributed phaseshift beamforming with the best result obtainable by installing repeaters, the technique presented in this paper is about three times better than the classic method. However, in cases where installing a repeater is an option, it is the preferred method.

The power of this method is due to the fact that it is scalable with the number of transmitters in each cluster. This paper presents only the case of four modules per cluster, because the algorithm that finds the optimal phase combinations [5] has (for the moment) exponential complexity and, in order to achieve results with a reasonable period of time and with reasonable power consumption, the number of devices must be limited. Work is in progress to provide a faster algorithm of cluster discovery algorithm for this technology.

The final note on the work presented in this paper is that intra-cluster communications have been totally ignored (with the exception of broadcasts - which are however necessary due to inter-cluster communication). The reason for this is to avoid creating interference between intra and inter cluster communications. Inter-cluster communication requires much greater resources, and must be protected better against interference. There are two ways to achieve this protection: by dividing the time interval into separate time zones or by using a new technique that we propose. From the point of view of time separation, there are two orthogonal time chunks for communication: an intercluster chunk and an intra-cluster chunk. It can be said that this paper details the inter-cluster chunk and ignores the other chunk. However, our team is fully confident that there is another solution: VCSMA/CA
(Virtual Carrier Sense Multiple Access With Collision Avoidance). This is work in progress, and it is based on the fact that the problem of interference has already been solved with individual devices by RTS/CTS mechanisms or by busy-tone mechanisms (BTMA - Busy Tone Multiple Access [2]). In our case, instead of individual transmitters we have virtual transmitters.

\section{Acknowledgements}

The research reported in this paper has been supported by the Ministry of Education, Youth and Sports of the Czech Republic under research program MSM 6840770014, and by the Grant Agency of the Czech Technical University in Prague, grant No. SGS11/158/OHK3/3T/13.

\section{References}

[1] H. Aghvami, M. Dohler, J. Dominguez. Link capacity analysis for virtual antenna arrays. In IEEE Vehicular Technology Conference, 2002.

[2] D. Kivanc-Tureli, P. Nehaben, U. Tureli. Effective channel utilization using the RI-BTMA protocol. In Military Communications Conference, $200 \%$. MILCOM 200\%. IEEE, 2007, pp.1-7.

[3] U. Madhow, R. Mudumbai, G. Barriac. On the feasibility of distributed beamforming in wireless networks. IEEE Transactions on Wireless Communications 6(4), 2007.

[4] A. Moucha, V. Cerny, J. Kubr. Distributed System for Beamforming. Patent Application, Reg. No. 2011-25254 / 2011-785, 2011.

[5] A. Moucha, J. Gattermayer. Cluster discovery in phase-shift beamformed ad-hoc and sensor networks. In Proceedings of the International Wireless Communications and Mobile Computing conference IWCM, IEEE, 2011.

[6] H. Poor \& co., H. Ochiai, P. Mitran. Collaborative beamforming for distributed wireless ad hoc sensor networks. IEEE Transactions on Signal Processing 53:4110-4124, 2005.

[7] S. Servetto, A. S. Hu. Optimal detection for a distributed transmission array. In IEEE International Symposium on Information Theory, 2003.

[8] G. Wornell, J. Laneman. Distributed space-timecoded protocols for exploiting co-operative diversity in wireless networks. IEEE Transactions on Information Theory 49(10):2415-2425, 2003. 